\title{
Compliance with pre-operative instructions for procedures with conscious sedation: a complete audit cycle
}

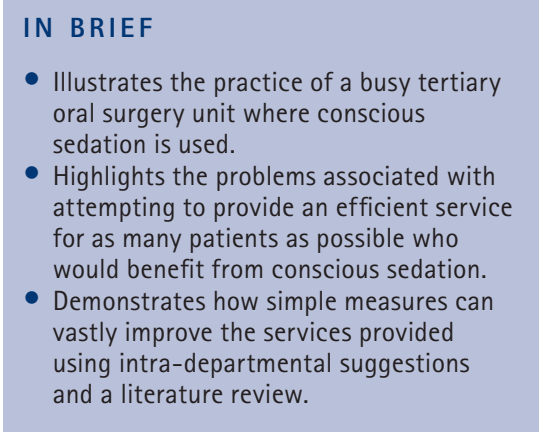

- Illustrates the practice of a busy tertiary oral surgery unit where conscious sedation is used.

Highlights the problems associated with attempting to provide an efficient service for as many patients as possible who would benefit from conscious sedation.

Demonstrates how simple measures can astly improve the services provided and a literature review.

\author{
P. Ravindra ${ }^{1}$ and C. Barrett ${ }^{2}$
}

VERIFIABLE CPD PAPER

\begin{abstract}
Introduction A wide variety of procedures in all surgical departments are performed under conscious sedation with midazolam. Patients are required to adhere to a list of pre-operative instructions but poor compliance has been noted anecdotally in our department and in the literature. This can lead to delayed and cancelled appointments. Aim We aimed to audit the compliance of patients in following preoperative sedation instructions in the oral and maxillofacial department of a large tertiary teaching hospital with a view to improving compliance. All patients undergoing conscious sedation for day case procedures in a five month period were audited. We implemented changes based on findings and a literature review, followed by a second eight month period of audit. Results Twenty-nine and 30 patients participated in each cycle respectively. In the first cycle over 55\% of patients were non-compliant. The most common reasons were not bringing a competent adult escort at the start of the appointment (17\% of total) and having consumed alcohol in the 24 hours pre-operatively (17\%). Based on this, the patient information leaflet was revised. Patients received copies when being listed and then with their posted appointment letter, as well as being verbally reminded before attending. Second cycle results revealed a $22 \%$ increase in compliance rates across all instructions with all patients bringing competent escorts along. Conclusions Efficacy within the NHS is often achieved with simple and practical improvement to clinical and administrative practice. By achieving a reduction in non-compliance rates, the department was able to reduce appointment delays and waiting lists, ultimately to the patients' benefit.
\end{abstract}

\section{INTRODUCTION}

Management of pain and anxiety is a vital part of dentistry. Almost half of all adults have been found to have moderate to extreme anxiety when attending their dentist. ${ }^{1}$ Most patients can be managed with behavioural techniques, but where these fail, it may be more appropriate to use conscious sedation (which may also reduce the need for general anaesthesia). ${ }^{2}$

In our unit, intravenous midazolam sedation is used to help manage anxious patients who are undergoing dentoalveolar surgery. Anecdotally, high

${ }_{1 *}$ Division of Gastrointestinal Surgery, School of Graduate Entry Medicine and Health Sciences, University of Nottingham Medical School at Derby, Royal Derby Hospital, Uttoxeter Road, Derby, DE22 3DT; ${ }^{2}$ Department of Oral and Maxillofacial Surgery, Queen's Medical Centre Campus, Nottingham University Hospitals, Derby Road, Nottingham, NG7 2UH

${ }^{*}$ Correspondence to: Dr Pravisha Ravindra

Email: pravisharavindra@doctors.org.uk;

Online article number E6

Refereed Paper - accepted 28 October 2011

DOI: 10.1038/sj.bdj.2012.99

${ }^{\circledR}$ British Dental Journal 2012; 212: E6 non-compliance rates with pre-operative instructions had been noted. This could lead to delayed and cancelled appointments, causing inconvenience and longer waiting lists, to the detriment of patients.

There are relatively few reports in the literature auditing the practicalities of using conscious sedation despite regular audit in this area being recommended. ${ }^{3}$

We aimed to audit patients' compliance with pre-operative instructions when attending for outpatient midazolam sedation for oral surgery procedures. The gold standard would be that all patients complied with all pre-operative instructions. Based on results, we aimed to determine ways of improving patient compliance and hence reduce wasted clinical time. We describe the completed audit cycle.

\section{MATERIALS AND METHODS}

\section{The situation before audit}

Patients who were assessed as being suitable for out-patient sedation in the department (generally ASA I or II) were given verbal and written instructions on the day of their assessment and placed on the waiting list. ${ }^{4}$ The existing patient information leaflet was evaluated and found to contain sixteen pre-operative instructions (see Table 1 where they are grouped by theme).

Patients subsequently received an appointment date by post and were asked to contact the department at their convenience via telephone in order to confirm attendance.

\section{Subjects and settings}

All patients attending for intravenous sedation in the Oral and Maxillofacial Department of the Queen's Medical Centre, Nottingham, between February and June 2008 were audited. Following implementation of changes, the same study was repeated between March and December 2009. A longer time frame was used in the second cycle in order to accumulate a comparable patient cohort; this was more difficult in the second cycle due to reduced conscious sedation sessions within the department. 


\section{Data collection}

Compliance with the 16 points on the instruction sheet was recorded by the oral surgeon or the nurse attending to the patient at their appointment. If the patient was non-compliant, the recorder was given the options 'forgot', 'didn't realise/know', 'unable to' and 'other' (where space was given to expand). Demographic information as well as time between listing for the procedure and the actual treatment date was recorded.

At the end of the first audit cycle findings were presented to and discussed within the department. Suggestions for improving the compliance rates were considered and changes implemented (see Discussion). Seven months were allowed for the changes to be fully integrated and the audit was then repeated using the same method as before (allowing for the new shortened list of pre-operative instructions). Results were compared with the original data to establish if the interventions had been successful.

\section{Analysis}

Analysis of results was undertaken using GraphPad Prism, version 5.00 for Microsoft Windows (GraphPad Software, San Diego, California, USA). The null hypothesis would be that there was no difference in compliance between the two cycles of audit. Data was tested for normality using the Kolmogorov-Smirnov test. Groups of non-parametric data were compared using the Mann-Whitney U test, while groups of parametric data were compared using the paired or unpaired t test, as appropriate. $\mathrm{P}$ values for categorical data were determined using the chi-squared test, while Fisher's exact test was utilised for smaller sample sizes. In all cases, $p$ had to be $\leq 0.05$ to be considered significant. Free text responses were summarised and reported where relevant.

\section{RESULTS}

\section{First cycle results}

In the first period audited, 29 patients attended appointments for oral surgery procedures under midazolam sedation. Of these, 19 (66\%) were female and 10 (34\%) were male with a median age of 35 (range 17-63). The average time between being listed for a procedure and the actual procedure date was 72 days (7-155 days).

Overall, 16 (55\%) patients were noncompliant in some way. Of these, 12 were women and 4 were men, but this was not a statistically significant difference $(p=0.270)$. There were a total of 27 incidences of non-compliance. There did not appear to be any age predilection $(p=0.125)$.

Non-compliance rates are illustrated in Figure 1 where pre-operative instructions have been grouped by theme. Most noncompliance was related to escorts and issues with dress (both seven incidences, 24\% of patients). Five (17\%) people had consumed alcohol in the 24 hours pre-operatively and there were five incidences (17\%) of consuming either food in the previous 3-4 hours or fluid in the previous 2 hours.

In more than half of all cases (52\%), the patient stated that their reason for noncompliance was that they did not know of a particular instruction, while 33\% of patients stated that they were 'unable' to comply.

More specifically, in all but one case, patients whose escort did not arrive with them at the start of the appointment stated they were unable to arrange it. All patients who consumed alcohol in the 24 hours preoperatively stated they were unaware of the rule. The majority of patients who had consumed fluids 2 hours pre-operatively had done so because they had forgotten, while all patients who wore earrings to the appointment were unaware of the rule against.

Finally, there was no significant link between the incidence of non-compliance and the time period between the booking appointment and the actual procedure $(p=0.934)$.

\section{Second cycle results}

Following the implementation of changes, compliance rates were re-audited. In the second period, 30 patients attended of which 20 (67\%) were female and 10 were male (33\%). The median age was 38 (range 22-61). The average time between being listed and the actual procedure was 52 days (7-134 days). This time, there was a weakly significant link between age and non-compliance ( $\mathrm{p}=0.05)$, whereby older patients were more likely to be non-compliant.

Ten patients (33\% of total) were noncompliant in some way. There were a total
Table 1 List of 16 points given on original patient information leaflet. Those highlighted in red remained in cycle 2 , after the implementation of changes. Those marked * were the instructions also emphasised in the appointment letter as well as by phone

\begin{tabular}{|l|l|}
\hline No. & Instruction \\
\hline \multicolumn{2}{|l|}{ Food c drink } \\
\hline $1^{*}$ & No food for 3-4 hours pre-op \\
\hline $2^{*}$ & No fluids for 2 hours pre-op \\
\hline Escort \\
\hline $3^{*}$ & Escort is a competent adult \\
\hline $4^{*}$ & $\begin{array}{l}\text { Escort arrives with patient at } \\
\text { start of appointment }\end{array}$ \\
\hline $5^{*}$ & $\begin{array}{l}\text { Escort is not responsible for anyone } \\
\text { other than patient, eg small children }\end{array}$ \\
\hline Transport \\
\hline 6 & $\begin{array}{l}\text { Arrangements in place for transport } \\
\text { home in a car or taxi }\end{array}$ \\
\hline Alcohol \\
\hline $7^{*}$ & No alcohol for 24 hours pre-operatively \\
\hline Dress & \\
\hline 8 & Avoid tight clothing around neck \\
\hline 9 & Avoid tight clothing around arms \\
\hline 10 & No contact lenses \\
\hline 11 & No lipstick \\
\hline 12 & No nail varnish or false nails \\
\hline 13 & No earrings \\
\hline 14 & No lip/tongue piercings \\
\hline Medication \\
\hline 15 & Regular medications taken \\
\hline 16 & $\begin{array}{l}\text { Brought regular medication with them } \\
\text { (if appropriate), eg GTN spray, inhalers }\end{array}$ \\
\hline
\end{tabular}

of 14 incidences of non-compliance. This represented an absolute decrease of $22 \%$ in non-compliant patients, as well as a relative reduction of $48 \%$ in non-compliant incidents. It is not, however, statistically significant $(p=0.100)$. There was no significant difference between the genders $(p=0.255)$. Compliance improved in all categories except alcohol consumption.

Non-compliance was mainly related to alcohol consumption in the 24 hours pre-operatively (six incidences, 20\% of total). This was followed by issues about dress (three incidences, 10\%). Finally there were two incidences of issues with starving guidelines (7\%) and two incidences of issues with medication (7\%). The 


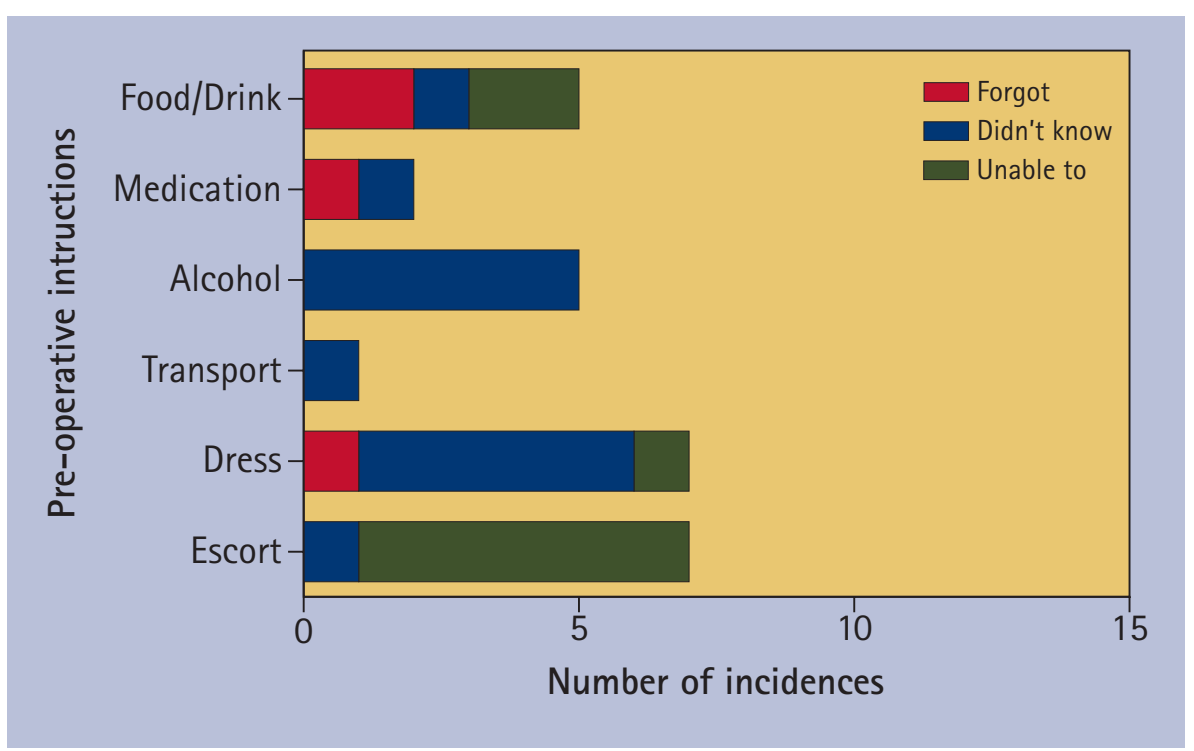

Fig. 1 Graph showing instructions that patients were non-compliant with and reasons for non-compliance

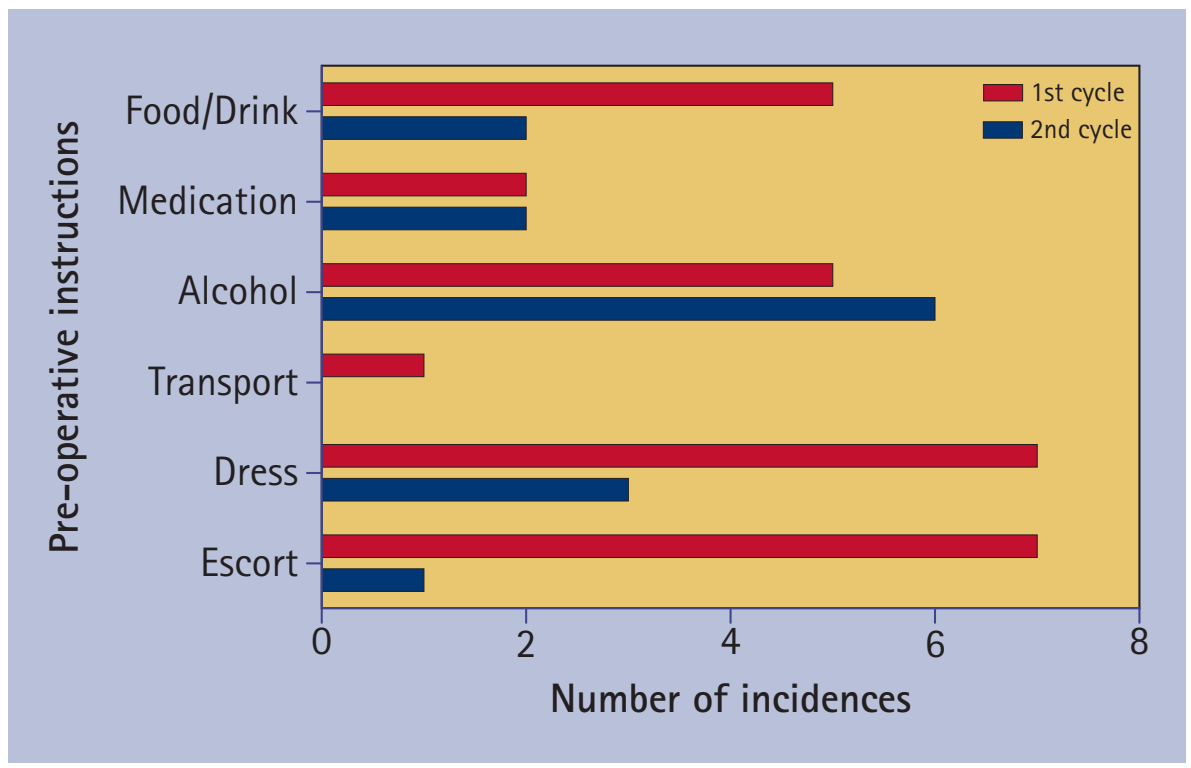

Fig. 2 Graph comparing incidences of non-compliance with pre-operative instructions in both cycles of audit

remaining non-compliant instructions are shown grouped by theme in Figure 2. It is apparent from this graph that in the categories which would have led to cancellation of the patient's appointment, starving and escort, non-compliance was reduced by more than $50 \%$. The same applied for transport and dress categories, which would have led to considerable delays in a patient's appointment.

Regarding reasons given for non-compliance, in half of all cases the patient stated they did not know of the particular instruction. Forty-three percent stated that they were aware but had forgotten, which was particularly the case with not consuming alcohol.
Finally, there again appears to be no significant link between the incidence of noncompliance and the time period between the booking appointment and the actual procedure ( $p=0.699)$.

\section{DISCUSSION}

Poor patient compliance with pre-operative instructions in day case procedures has been widely documented across several areas of medicine. Before this audit, anecdotally there was a high non-compliance rate with pre-operative sedation instructions given to patients in the department which could lead to delayed and cancelled appointments. We aimed to improve compliance and reduce wasted clinical time.
The most recent guidance on the use of conscious sedation in dental practice was published by the Standing Dental Advisory Committee (SDAC) for England and Wales in $2003,{ }^{5}$ and in Scotland in 2006. ${ }^{10}$ These have been adopted by the General Dental Council as the best practice standards by which conscious sedation is practised in the UK. ${ }^{6}$ It is recommended that patients receive careful verbal and written instructions regarding the effects of sedation and their responsibilities both before and immediately after the procedure. ${ }^{5}$

\section{First cycle}

The first cycle of this audit established the actual level of non-compliance with the existing list of pre-operative instructions that had been used in the department.

The standard of a $100 \%$ compliance rate with pre-operative instructions was not met, with the non-compliance rate of 55\% being a particular concern. Forty percent of all incidents of non-compliance were with instructions which would have led to cancellation of the appointment, while $28 \%$ of incidents would have led to delays in the starting time.

\section{Implementation of changes}

Following the first audit cycle, the literature was reviewed and possible changes to improve compliance considered.

Many factors including age, ethnicity, race, distance from home and time between booking appointment and procedure date have been shown in previous studies to contribute towards non-compliance with pre-operative instructions. ${ }^{7,8}$ Reviews into the most effective ways of delivering preoperative information have found the most widely used are pamphlets. ${ }^{9}$

An especially worrying finding was that $24 \%$ of patients were attending without appropriate escort arrangements in place. These patients all stated that they were unable to arrange it. This may be due to them not realising the importance of arriving at the start of the appointment with an escort. Other studies have found that often patients do not comply due to difficulty understanding the reasons behind instructions. ${ }^{8,10}$

In this audit, the main reason given by patients for non-compliance was being unaware of a particular rule, despite the fact that all patients had been given 
information verbally and in leaflet form. It could be that patients were, in fact, aware of the rule but denied knowledge as they were afraid of being refused the planned treatment. It also seemed possible that the period of more than 2 months between patients being given instructions and their actual appointment would affect their compliance. ${ }^{7}$ It was therefore decided that two additional reminders of the instructions considered to be most important would be given to patients nearer to their appointment time.

The next most common reason given was patients claiming they were unable to comply with a particular instruction even though they were aware of it. It was likely here, that the extensive list of instructions given to patients might have led to major requirements being overlooked among the minor ones. Therefore we decided to simplify the original pre-operative instructions, while still emphasising essential safety points.

The literature, including guidance documents, ${ }^{5,11}$ was considered before altering the instructions and key points discussed below.

\section{Escort}

Departmental guidance is in line with national guidance that a responsible adult escort must accompany the patient home and assume responsibility for the rest of the day. The patient should not care for young children or dependent adults on the day. The escort must acknowledge responsibility of this before the appointment. Transport home should be arranged by car or taxi. ${ }^{12}$ Therefore none of these specific instructions were left out of the new set of patient instructions.

\section{Fasting}

There is debate as to whether fasting is normally required before undergoing conscious sedation. ${ }^{13,14}$ Some schools of thought uphold that the same rules apply for conscious sedation as for general anaesthesia, due to possible depression of upper airway reflexes. ${ }^{15}$ This is most relevant in emergency situations where there is a risk of pulmonary aspiration of gastric contents. However, Green et al. found in emergency department procedures involving intravenous sedation and analgesia, there were no reported cases of aspiration in the literature. ${ }^{16} \mathrm{~A}$ recent study into fasting before conscious sedation for dental treatment has in fact shown that the majority of patients report experiencing adverse symptoms such as increased anxiety as a result. ${ }^{17}$ Recently published guidance from NICE has recommended that fasting is not required in young people when verbal contact is maintained during the sedation. ${ }^{18}$

This Department was, however, bound by hospital guidance at the time of the audit and therefore there was no change in the fasting guidance given.

\section{Pre-operative alcohol consumption}

There is evidence to suggest that alcohol induces CYP450 3A4 induction thus increasing plasma midazolam concentration and prolonging sedative effects. ${ }^{19}$ Therefore some authors recommend no alcohol on the day of the visit. ${ }^{20}$ The department chose to extend the non-alcoholic period to 24 hours before the appointment to ensure patients did not consume large amounts of alcohol the night before.

\section{Dress code}

There is no specific guidance regarding clothing requirements for patients undergoing sedation. However, for practical reasons, it was initially suggested that patients wore loose clothing and removed earrings etc. On review of the pre-operative instructions, it was felt some of these additional requirements would be ideal, but did complicate the patient instructions. They were therefore dropped in an attempt to improve compliance with the more essential points.

Taking into account the above, a new shortened list of pre-operative instructions was implemented before cycle two of the audit. Figure 1 shows these points (in red).

Overall, the main recommendations for change after the first audit cycle were:

1. To simplify the current patient information leaflet as well as to improve layout and include concise wording

2. To supplement the leaflet with additional reminders about the essential instructions (shown with * Table 1) on two occasions: in writing with their appointment date, and verbally one week pre-operatively over the telephone.

\section{Second cycle}

Following implementation of the changes described, overall compliance rates increased from $45 \%$ to $67 \%$.

Rates of non-compliance with starving instructions, escort and transport issues all fell by more than $50 \%$. We attribute the fall in non-compliance with dress issues to the revised instruction regime.

Of all of the incidences of non-compliance in the second cycle, failing to abstain from alcohol consumption pre-operatively accounted for almost half. This may be explained by the fact that this patient group are inherently very anxious regarding attendance and were 'self medicating' despite being aware that they had been instructed to abstain from alcohol. In practice, however, this was dealt with on a case by case basis, by exploring with the patient exactly how much alcohol was consumed and when, allowing the oral surgeon to decide if the appointment would need to be rescheduled.

\section{Limitations}

This two-cycle audit is not without its limitations. The main issue with the significance of our results would be the sample size used. This is, however a reflection of the size of the patient base being analysed. Nonetheless, it is an important factor to take into account. Secondly, there is always the possibility that patients may have deliberately concealed some elements of non-compliance, for example if they had consumed food/fluids/alcohol within the time stated. This may be more so if they felt that they may not receive the care that they had expected that day.

\section{CONCLUSION}

Overall, changes implemented after the first audit cycle have been relatively successful in achieving the aims of this audit; improving patient compliance with most pre-operative instructions and reducing wasted clinical time, thus achieving greater efficacy with NHS resources and raising Departmental standards. This is encouraging as significant resources were not required.

Based on the results of the second cycle, the following recommendations are made:

1. To review the requirement to avoiding alcohol in the 24 hours before the procedure and carry out a more 
detailed audit on compliance with this

2. To review the starving guidelines used for conscious sedation in the department

3. To alter the reminder phone call to a period within 3 days of the procedure to increase information retention rates and improve compliance.

Competing interests: None.

Provenance: this article is the sole work of the named authors.

Funding: none.

Previous presentation: this work was previously the subject of a poster presentation at the Association of Surgeons of Great Britain and Ireland conference in Liverpool, 2010.

1. Department of Health. Adult dental health survey 2009. London: The NHS Health and Social Care Information Centre, 2011.

2. Donaldson L, Wild R. A conscious decision: review of the use of general anaesthesia and conscious sedation in primary dental care. London: Department of Health, 2000.

3. Girdler N, Wilson K E. Overview of conscious sedation for primary dental care. Faculty Dent J 2010; 1: 99-102.

4. American Society of Anesthesiologists. 2011 Relative Value Guide. Park Ridge, IL: ASA, 2011.

5. Standing Dental Advisory Committee. Conscious sedation in the provision of dental dare: report of an expert group on sedation for dentistry. London: Department of Health, 2003

6. General Dental Council. Standards for Dental Professionals. London: General Dental Council, 2005.

7. Kaye J D, Richstone R, Cho J S, Tai J Y, Arrand J, Kavoussi L R. Patient noncompliance before surgery. BJU International 2010; 105: 230-233.

8. Laffey J G, Boylan J F. Patient compliance with preoperative day case instructions. Anaesthesia 2001 56: 906-924.

9. Hodgkinson B, Evans D, O'Neill S. Knowledge retention from pre-operative patient information: a systematic review. Adelaide: The Joanna Briggs Institute for Evidence Based Nursing and Midwifery, 2000.

10. Laffey J G, Carroll M, Donnelly N, Boylan J F. Instructions for ambulatory surgery-patient comprehension and compliance. Ir J Med Sci 1998; 167: 160-163.

11. Scottish Dental Clinical Effectiveness Programme. Conscious sedation in dentistry: dental clinical guidelines. Dundee: SDCEP, 2006:

12. Standing Dental Advisory Committee. Conscious sedation in the provision of dental care: new guidelines. SAAD Dig 2004; 21: 20-22.

13. McKenna G, Manton S. Pre-operative fasting for intravenous conscious sedation used in dental treatment: are conclusions based on relative risk management or evidence? Br Dent J 2008; 205: 173-176.

14. Standing Dental Advisory Committee. General anaesthesia, sedation and resuscitation in dentistry. In The Poswillo report. London: Department of Health, 1990.

15. Murphy P J, Erskine R, Langton J A. The effect of intravenously administered diazepam, midazolam and flumazenil on the sensitivity of upper airway reflexes. Anaesthesia 1994; 49: 105-110.

16. Green $\mathrm{S} M$, Krauss B. Pulmonary aspiration risk during emergency department procedural sedation - an examination of the role of fasting and sedation depth. Acad Emerg Med 2002; 9: 35-42.

17. McKenna G, Manton S, Neilson A. A study of patient attitudes towards fasting before intravenous sedation for dental treatment in a dental hospita department. Prim Dent Care 2010; 17: 5-11.

18. National Institute for Health and Clinical Excellence. Sedation for diagnostic and therapeutic procedures in children and young people. CG112. London: NICE, 2010.

19. Riss J, Cloyd J, Gates J, Collins S. Benzodiazepines in epilepsy: pharmacology and pharmacokinetics. Acta Neurol Scand, 2008; 118: 69-86.

20. Girdler N M, Hill C M, Wilson K E. Clinical sedation in dentistry. London: Wiley-Blackwell, 2009. 